\title{
RADAR REMOTE SENSING
}

\section{ACOUSTIC NOISE ATMOSPHERIC RADAR WITH NONPARAMETRIC COPULA BASED SIGNAL PROCESSING}

\author{
R.B. Sinitsyn E F.J. Yanovsky" \\ National Aviation University, Radio-Electronics Department \\ 1, Komarova ave., Kiev, 03058, Ukraine \\ •Address all correspondence to F.J. Yanovsky E-mail: felix.yanovsky@ieee.org \\ In this paper we discuss a new approach to creating the acoustic atmospheric radar (sodar) \\ which is based on noise sounding waveform. Such sounding waveform has extremely high \\ range and speed resolution. These good properties exist only in the case when we have \\ independent samples, and a noise signal forms such independent samples because of its nature. \\ The prototype of the radar is described and investigated theoretically, using computer \\ simulation and in natural conditions. Non-parametrical signal processing algorithm is \\ developed based on the statistical approach and digital signal processing. It has wonderful \\ properties of invariance to the group of the noise and signal transforms and stable level of the \\ false alarm probability. The algorithm can be applied for both acoustic and microwave noise \\ radars. The use of the FFT algorithm increases the speed of calculations and gives us the \\ possibility of constructing digital random signal radars. These new properties allow us to \\ simplify signal detection algorithms and measure a distance, an azimuth and a target velocity \\ simultaneously with high resolution and accuracy.
}

KEY WORDS: radar sensing, signal processing, noise signal

\section{INTRODUCTION}

\subsection{Noise Radars}

A possibility of noise signal application in radar was widely discussed in the 70-s of the XX century. A new impulse to real noise radar development was given in the first decade of the XXI century [1] though random noise radar which transmits a CW Gaussian true random noise signal was described by [2]. This radar system has been used to estimate the Doppler spectra of moving objects.

As Doppler spectra are the most important source of information in Doppler radar used for weather observations [3], it is interesting to investigate noise radar as a tool for remote sensing of the atmosphere. 
The potential of noise radar for weather applications was considered in [4] where it was shown that an impressive advantage of noise radar is its possibility to measure very wide Doppler spectra without uncertainty in distance, but the retrieval of meteorological information from returns using noise radar is rather a complicated application of such radar. One of the conclusions was that additional research work and technological efforts should be done to achieve necessary clarity for retrieving weather information with the help of noise radar.

\subsection{Sodar}

Sodar is an abbreviation similar to radar (SOnic Detection And Ranging). Sodar is used for applications in the atmosphere in contrast to sonar that is in fact a hydroacoustic radar. Acoustic devices are much cheaper and more accessible for experiments than microwave radar. Sodars are normally used for remote measuring of the vertical turbulence structure and the wind profile of the lower layer of the atmosphere. Sodar systems usually use an ordinary sine wave as a transmitted signal. The possibility of using noise signal in acoustic radar was considered by [5]. The likelihood ratio has been obtained for the noise signal in that paper using kernel estimation of probability density functions (PDF).

\subsection{Purpose of this Paper}

Noise signal processing algorithms for radars and sodars were considered by [6]. Both parametrical and non-parametrical algorithms were developed and analyzed in that paper. The disadvantage of the parametrical algorithm was its obvious idealism connected with the supposition that a signal received corresponds to the accepted model while it may be different in reality, decreasing the efficiency of the algorithm sharply. A shortcoming of the developed nonparametric algorithm was its complexity related to the necessity to estimate the conditional distribution function of a signal received.

This paper aims to continue the development of the statistical approach to synthesizing algorithms for digital signal processing in noise radar and sodar and create a prototype of the noise radar (sodar) using the software radar concept.

A feature of the approach accepted in this paper is the development of nonparametric copula based detection algorithms, which allows us to avoid estimating conditional distribution function in order to simplify the implementation of nonparametric algorithms. Another feature is the fact that the case of Multiple Input Multiple Output (MIMO) that promises significant improvement of performance characteristics is also considered here.

The area of this research is limited to the noise or the random signal radar (sodar) with digital signal processing and statistical signal processing algorithms. Nonparametrical approach is used with the application of: 1) kernel estimates of PDF of signals and clutters, and 2) copula-based detection algorithms. 
In the framework of this approach, Monte-Carlo simulation for the suggested algorithm is done and experimental check based on the developed acoustic physical model is provided, using the developed noise sodar prototype.

\section{STATISTICAL SYNTHESIS OF SIGNAL PROCESSING ALGORITHM}

\subsection{Basic Signal Models}

Let us the sounding signal is $\mathbf{x}=\left\{x_{1}, x_{2}, \ldots, x_{n}\right\}$ and the sum of the reflected signal and a receiver noise is $\mathbf{y}=\left\{y_{1}, y_{2}, \ldots, y_{n}\right\}$. In case when a signal corresponds to white noise model the mutual PDF of sounding and reflected signals is

$$
f_{x y}(\mathbf{x}, \mathbf{y})=\prod_{i=1}^{n} f_{x y}\left(x_{i}, y_{i}\right)=\prod_{i=1}^{n} f_{x}\left(x_{i}\right) f_{y}\left(y_{i} \mid x_{i}\right)
$$

It is a general signal model for one channel, the PDF can be written as a product of univariate PDF (in the case of absence of the useful signal or if the delay does not correspond to the distance to the target):

$$
f_{x y}(\mathbf{x}, \mathbf{y})=\prod_{i=1}^{n} f_{x}\left(x_{i}\right) f_{y}\left(y_{i} \mid x_{i}\right)=\prod_{i=1}^{n} f_{x}\left(x_{i}\right) f_{y}\left(y_{i}\right) \text {. }
$$

For MIMO system, the mutual PDF of the sounding and reflected signals can be represented as

$$
f_{x y}^{m}(\mathbf{x}, \mathbf{y})=\prod_{k=1}^{m} \prod_{j=1}^{m} \prod_{i=1}^{n} f_{x y}\left(x_{i j}, y_{i k}\right)=\prod_{k=1}^{m} \prod_{j=1}^{m} \prod_{i=1}^{n} f_{x}\left(x_{i j}\right) f_{y}\left(y_{i k} \mid x_{i j}\right)
$$

in case of presence of a useful signal; and

$$
f_{x y}^{m}(\mathbf{x}, \mathbf{y})=\prod_{k=1}^{m} \prod_{j=1}^{m} \prod_{i=1}^{n} f_{x y}\left(x_{i j}, y_{i k}\right)=\prod_{k=1}^{m} \prod_{j=1}^{m} \prod_{i=1}^{n} f_{x}\left(x_{i j}\right) f_{y}\left(y_{i k}\right)
$$

in case of no signal. In the last two formulas, $m$ is the number of receivers and transmitters for multichannel case.

\subsection{Functional Transform}

We can transform the vector $(x, y)$ to a new random variable $\left(x_{T}, y_{T}\right)$, using two marginal cumulative distribution functions $x_{T}=F_{x}(x), y_{T}=F_{y}(y)$ as functional 
transforms. The transformed vector $\left(x_{T}, y_{T}\right)$ has uniform distribution, if random variables $x$ and $y$ are independent. It is a functional transform to [0,1] space, and it is an identical transformation, that is, no information losses (the true distribution functions are used here).

\subsection{Copula}

The bivariate cumulative distribution function $C\left(x_{T}, y_{T}\right)$ of the transformed variables $\left(x_{T}, y_{T}\right)$ is called a copula of these variables; and according to Sklar's theorem

$$
F(x, y)=C\left(F_{x}(x), F_{y}(y)\right),
$$

where $F(x, y)$ is a bivariate cumulative distribution function of $(x, y)$. This property of copula is used in our approach to simplify signal processing algorithm.

Let us consider copula density for our transformed variables. The density function, corresponding to the copula $C\left(x_{T}, y_{T}\right)$ is a derivative

$$
c\left(x_{T}, y_{T}\right)=\frac{\partial C\left(x_{T}, y_{T}\right)}{\partial x_{T} \partial x_{T}} .
$$

So, density distribution is substituted by the derivative of the copula. If a useful signal is absent the copula density function has a uniform distribution on $[0,1]^{2}$. But if a useful signal is present the copula density function has some other distribution on $[0,1]^{2}$.

Mathematically, copula density for signal model after functional transform is

$$
f_{x_{T} y_{T}}^{m}\left(\mathbf{x}_{T}, \mathbf{y}_{T}\right)=\prod_{k=1}^{m} \prod_{j=1}^{m} \prod_{i=1}^{n} f_{x_{T} y_{T}}\left(x_{i j T}, y_{i k T}\right)=\prod_{k=1}^{m} \prod_{j=1}^{m} \prod_{i=1}^{n} c\left(x_{i j T}, y_{i k T}\right) .
$$

In case of zero hypothesis (No signal), it is a uniform distribution because of the identical transformation $\left(x_{T}, y_{T}\right)$ through the cumulative distribution function applied.

\subsection{Neyman-Pearson Copula Decision Rule}

The detection procedure is based on testing the hypotheses about the density function of transformed signals on the basis of Neyman - Pearson criterion.

The copula likelihood function

$$
l\left(\mathbf{x}_{T}, \mathbf{y}_{T}\right)=\prod_{k=1}^{m} \prod_{j=1}^{m} \prod_{i=1}^{n} c\left(x_{i j T}, y_{i k T}\right)
$$


is compared with a threshold.

Actually, this is a transform of likelihood function using true cumulative distribution function, that is, sufficient statistics. Copula here is a theoretical, true copula (not an estimate). That is why it is a parametrical algorithm. Nonparametrical properties will appear only after the substitution of copula estimate later on.

\subsection{Estimates of distribution functions}

Let us replace the cumulative distribution functions $F_{x}(x)$ and $F_{y}(y)$ by their estimates $\hat{F}_{x}(x)$ and $\hat{F}_{y}(y)$. It is assumed, that if the size of a sample is increased, the estimate converges to a cumulative distribution function.

Transformations of the sounding and reflected signals using the estimates

$$
x_{T i}=\hat{F}_{x}\left(x_{i}\right), \quad y_{T i}=\hat{F}_{y}\left(y_{i}\right)
$$

will be used later.

The next step is to get the kernel estimate of a bivariate copula density function (in case of presence of signal)

$$
\hat{c}\left(x_{T}, y_{T}\right)=\sum_{i=1}^{n} K_{i}\left(x_{T}, y_{T}\right)
$$

where $n$ is the sample size, $K_{i}\left(x_{T}, y_{T}\right)=\frac{1}{n} \omega\left(x_{T}-x_{T i}, y_{T}-y_{T i}\right)$ is the kernels of the estimate, $\omega\left(x_{T}, y_{T}\right)$ is some probability density, $\left(x_{T i}, y_{T i}\right)$ is the sample unit $i$, which is the basis for an estimate.

This step gives us a possibility to implement learning by instruction using high enough sample size (about 40000). To estimate $\hat{F}_{x}(x)$ of one-dimensional cumulative distribution function the kernels look as the following integral of the kernel for density:

$$
P_{i}(x)=\frac{1}{n} \int_{-\infty}^{x} \int_{-\infty}^{\infty} \omega\left(u-x_{i}, v-y_{i}\right) d u d v
$$

The copula density function is replaced by its kernel estimate

$$
\lambda_{T}\left(\mathbf{x}_{T}, \mathbf{y}_{T}\right)=\prod_{i=1}^{n} \hat{c}\left(x_{T i}, y_{T i}\right) \text { with } \hat{c}\left(x_{T i}, y_{T i}\right)=\sum_{j=1}^{n} K_{j}\left(x_{T i}, y_{T i}\right) \text {. }
$$


This is for a single-channel case. Logarithm of the likelihood function is

$$
\lambda_{T}\left(\mathbf{x}_{T}, \mathbf{y}_{T}\right)=\sum_{i=1}^{n} \ln \left(\hat{c}\left(x_{T i}, y_{T i}\right)\right)
$$

and for MIMO variant it is

$$
\lambda_{T}\left(\mathbf{x}_{T}, \mathbf{y}_{T}\right)=\sum_{k=1}^{m} \sum_{j=1}^{m} \sum_{i=1}^{n} \ln \left(\hat{c}\left(x_{T i j}, y_{T i k}\right)\right.
$$

\subsection{Decision Making and Statistical Modeling}

To make a decision, the likelihood function $\lambda_{T}\left(\mathbf{x}_{T}, \mathbf{y}_{T}\right)$ is compared with invariable threshold $C$.

Statistical Monte-Carlo modeling was done for single-channel case. Figure 1 presents the modeled detection characteristics.

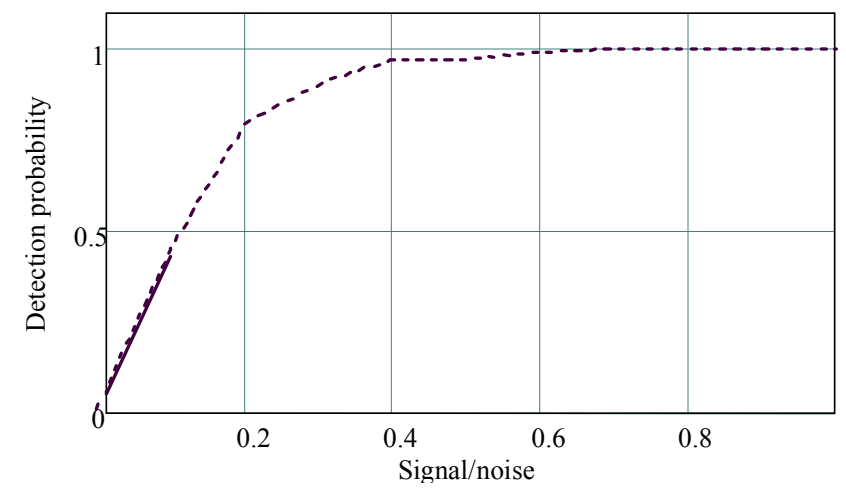

FIG. 1: Performance of detection. Solid curve - parametric algorithm, dashed curve nonparametrical algorithm $\alpha=0.01, N=100$

They are the dependence of the detection probability ( $y$ axis) on SNR - signal-tonoise ratio ( $x$ axis) for the classical parametrical algorithm (solid curve) and for the developed copula based nonparametrical algorithm (dashed curve). The calculation were done at sample size $N=100$ and false probability $\alpha=0.01$ (at least when SNR > 0.15). 


\section{EXPERIMENT}

In order to test the properties of the detection algorithms, the physical model of the noise sodar was developed and implemented. The low signal frequency and the low sound speed allows us to test in the practical experiment all the detection algorithms suggested using an ordinary computer as a signal processor.

The experimental system consists of an acoustic antenna with the FX-308B high frequency piezoelectric loudspeaker as a sounder and another acoustic antenna with a microphone as a receiver. The experimental sodar is shown in Fig. 2. The antennas are offset type parabolic reflectors. Also we have tried to use another FX-308B loudspeaker as a microphone. For generating sound signals, receiving and processing them we used an ordinary computer with a sound card and a low frequency amplifier for the sound card signal amplification.

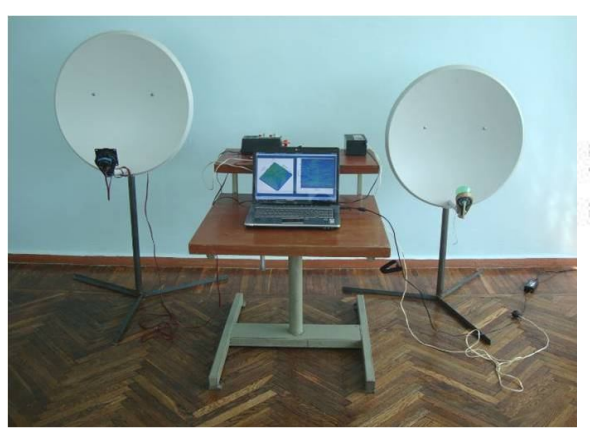

FIG. 2: The experimental sodar

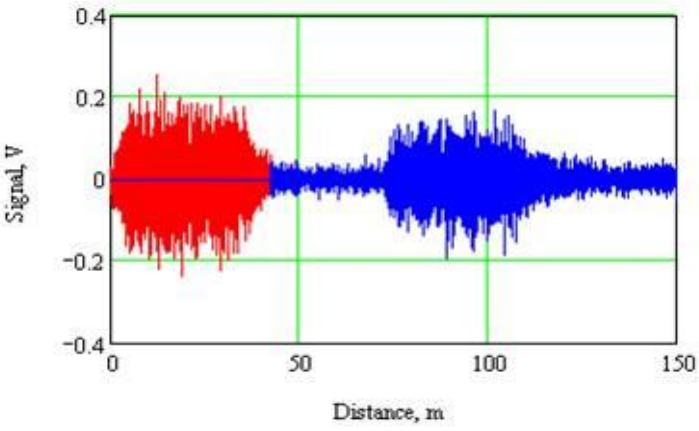

FIG. 3: The sounding (on the left) and reflected (on the right) noise pulses

The sampling frequency of the sounding noise signal is equal to $44100 \mathrm{~Hz}$. The duration of the noise pulse was $250 \mathrm{~ms}$. The sounding waveform and the reflected signal are shown in Fig. 3 in case of reflection from the building.

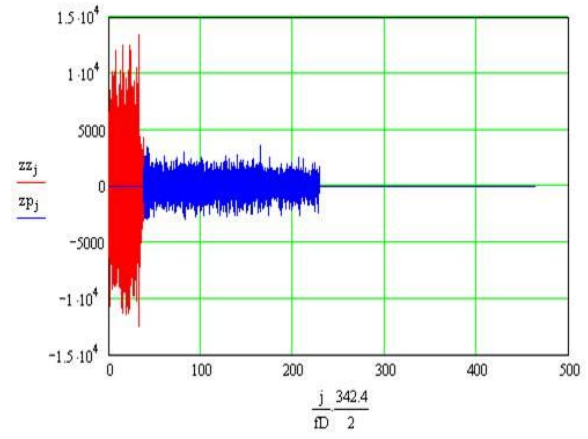

FIG. 4: The sounding waveform and returns from the atmosphere

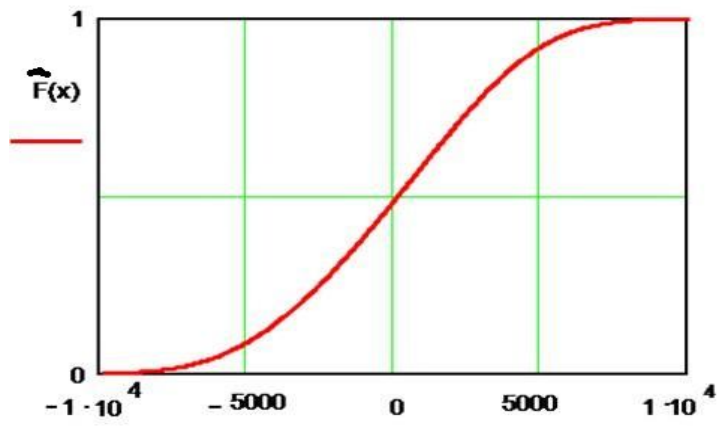

FIG. 5: Kernel estimate of the marginal cumulative distribution as functional transform function 
The sounding (on the left) and reflected (on the right) noise pulses in case of the slant sounding to the atmosphere are shown in Fig. 4.

The kernel estimate of the marginal cumulative distribution function is presented in Fig. 5. Processing was done for 100 samples of the sounding signal of the experimental acoustic radar. Sixteen-digit ADC was used, and $2^{16}$ corresponds to 1 Volt.

The decision function values in coordinates range - velocity as contour diagram is given in Fig. 6. Processing was done in accordance with the following expression

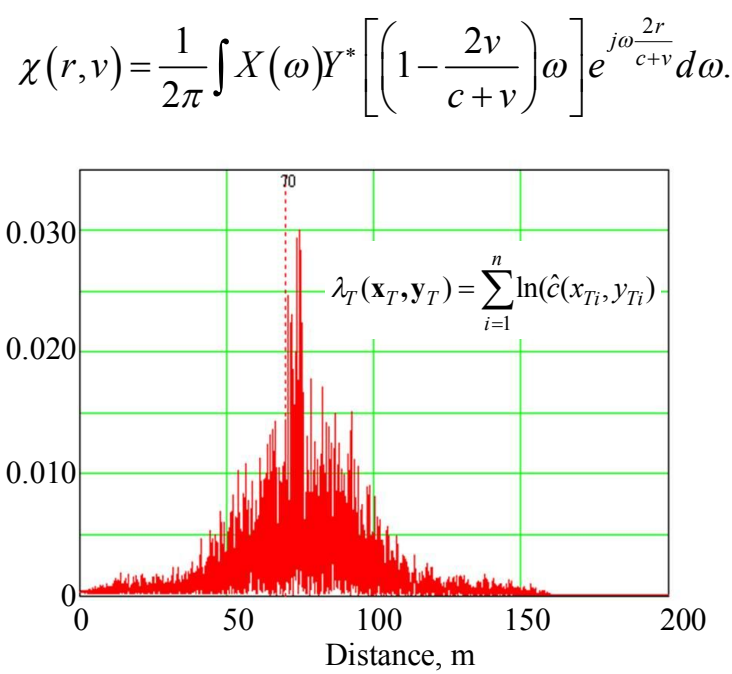

FIG. 6: Decision function. Dependence on distance (building at $70 \mathrm{~m}$ )

A result of atmosphere sounding is presented in Fig. 7. It can be interpreted as wind profile but azimuth resolution is not sufficient in case of single channel sodar with a small radiator.

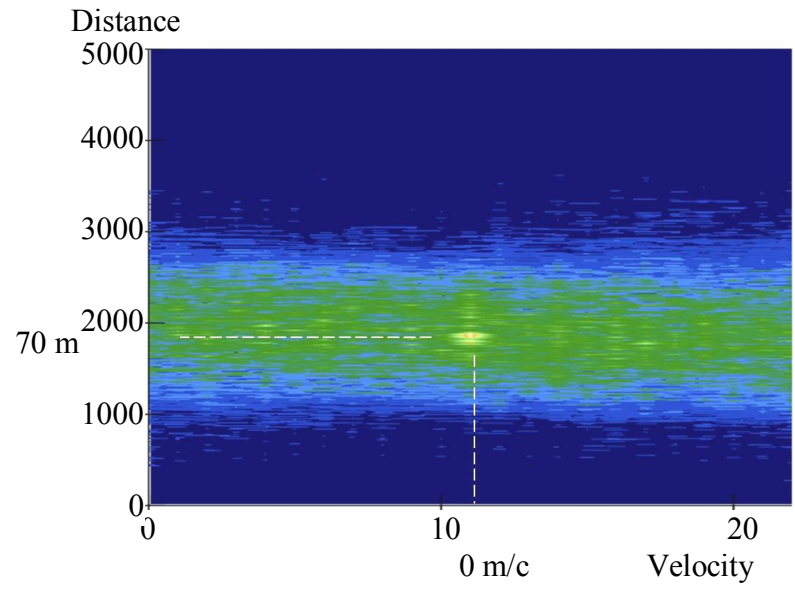

FIG. 7: Stable target at $70 \mathrm{~m}$ distance 


\section{CONCLUSIONS}

The noise signal sodar (radar) has remarkable properties based on the random nature of the sounding waveform. Application of copula based signal processing allows simplifying signal detection algorithms. Nonparametric algorithms have wonderful properties of invariance to the group of the noise and signal transforms and a stable level of the false alarm probability.

Angular resolution is not good enough in case of single-channel radar and smallsize antenna. Application of MIMO approach and algorithm allows to solve this problem. Independence of the noise signal allows us to simplify MIMO approach to radar design.

\section{REFERENCES}

1. Lukin, K., (2002), First International Workshop on the Noise Radar Technology (NRTW 2002), Yalta.

2. Narayanan, R.M. et al., (1997), Doppler estimation using a coherent ultra-wideband random noise radar. Proc, SPIE Conference on Radar Processing, Technology, and Applications, 3161:70-76.

3. Doviak, R. and Zrnic, D., (1993), Doppler radar and weather observations, Academic Press.

4. Yanovsky, F., (2002), Potential of a noise radar for meteorological applications, Proc. of the First Int. Workshop on the Noise Radar Technology, Yalta, pp. 195-200.

5. Sinitsyn, R., Beletsky, A., and Yanovsky, F., (2003), Noise signal for sodar applications, Proc. of Int. Conf. on the Noise Radar Technology, Kharkov, Ukraine, published in "Applied Radio Electronics", 4(1):107-110.

6. Yanovsky, F. and Sinitsyn, R., (2006), Ultrawideband Signal Processing Agorothms for Radars and Sodars, Proc. 3d International Conference on Ultrawideband and Ultrashort Impulse Signals, Sevastopol, Ukraine, pp. 66-71.

7. Gijbels, I. and Mielniczuc, J., (1990), Estimating the density of a copula function, Comm. Stat. Theory Methods. 19(2):445-464.

Volume 71, Number 4, 2012 